\title{
Self-organization of In nanostructures on Si surfaces
}

\author{
Maojie Xu, Arifumi Okada, Shoji Yoshida, and Hidemi Shigekawa ${ }^{a}$ \\ Institute of Applied Physics, CREST-JST, University of Tsukuba, Tsukuba 305-8573, Japan
}

(Received 30 December 2008; accepted 29 January 2009; published online 18 February 2009)

\begin{abstract}
Methods of forming various In nanostructures on Si surfaces are demonstrated. Using a high-index $\mathrm{Si}(311)$ surface, isolated nanotriangles and wires were grown by optimizing the deposition rate and substrate temperature. In contrast, nanodots were formed by the deposition of In on a $\mathrm{Si}(111)-\mathrm{In}-\sqrt{31} \times \sqrt{31}$ surface at room temperature $(\mathrm{RT})$ deposition. On a $\mathrm{Si}(111)-\mathrm{In}-4 \times 1 / \sqrt{31}$ $\times \sqrt{31}$ coexisting surface, nanowires were selectively grown in the $\mathrm{Si}(111)-\mathrm{In} 4 \times 1$ area by RT deposition through the nucleation promoted by the boundary barrier produced by the surrounding $\sqrt{31} \times \sqrt{31}$ area. Details were studied using scanning tunneling microscopy. (C) 2009 American Institute of Physics. [DOI: 10.1063/1.3085960]
\end{abstract}

Nanometer-sized low-dimensional structures, such as nanowires and nanodots, have been attracting growing interest because they provide typical systems for exploring interesting physical phenomena, such as quantized conductance, charge density wave, metal-insulator transition (MIT), and quantum confinement in reduced dimensions. Technologically, they also have great potential for application, for example, in optoelectronic and nanoelectronic devices. Among various methods employed to fabricate nanoscale structures, self-organization or self-assembly processes on material surfaces are used to create large-scale arrays of nanostructures. For example, strain in lattice-misfit heteroepitaxial growth gives rise to the self-organization of ordered arrays of semiconductor quantum wires and dots with uniform size and shape. ${ }^{1-3}$ Reconstructions, dislocations, steps, and facets also induce selective growth on a substrate surface. $^{4-6}$

Indium (In) is an attractive material widely used in semiconductor technologies as well as in fundamental studies. The growth of 1.0 ML of In on a Si(111) surface, for example, forms a self-assembled In nanowire array, In- $(4 \times 1)$. It is a typical quasi-one-dimensional (quasi-1D) metal system that induces MIT. $^{7}$ A recent study has revealed that the MIT of this system can be well controlled by optical doping, ${ }^{8}$ making this system a more applicable nanoscale building block that can controllably transport electrical charges. Owing to its anisotropic structure, the In- $(4 \times 1)$ surface has been used as a template for the growth of nanostructures. Self-organized $\mathrm{Pb}$ islands of uniform width and elongated shape were grown on the surface by taking advantage of substrate-mediated anisotropic strain relaxation. ${ }^{9}$ Adsorbed Co atoms showed a self-alignment on the surface by quasi-1D electron-gas-mediated interactions. ${ }^{10}$ The epitaxial Ag film on In- $(4 \times 1)$ surface formed a well-ordered stripe superstructure. ${ }^{11}$

However, only a uniform wire structure has been reported, and the method, for example, of forming isolated In nanowires or In nanodots on a Si surface has not been realized despite the interest of their applications. For more effective applications of the various attractive characteristics of In/Si system, development of the methods that enable the fabrication of In nanostructures in more applicable forms is

\footnotetext{
${ }^{a)} \mathrm{URL}:$ http://dora.ims.tsukuba.ac.jp.
}

required. In this letter, we present the methods we found for forming various In nanostructures on $\mathrm{Si}$ surfaces.

Experiments were carried out in an ultrahigh vacuum system with a base pressure of $1.5 \times 10^{-8} \mathrm{~Pa}$. Clean $\mathrm{Si}(111)$ and $\mathrm{Si}(311)$ surfaces were prepared as substrates by standard procedures. A heat-resistant evaporator was used to deposit In atoms at a flux of $0.3-6.0 \mathrm{ML} / \mathrm{min}$. Scanning tunneling microscopy (STM) images were taken at room temperature (RT).

First, we show the methods of forming isolated nanotriangles and wires. After preparing a clean $\mathrm{Si}(311)$ surface with a $3 \times 2$ structure, ${ }^{12,13}$ the deposition of more than $1 \mathrm{ML}$ of In on the surface at $\sim 300{ }^{\circ} \mathrm{C}$ formed a homogeneous $\mathrm{Si}(311)-\mathrm{In}-3 \times 2$ structure, as shown in Figs. 1(a) and 1(b). On the other hand, for a substrate temperature lower than $50{ }^{\circ} \mathrm{C}$, a nanoscale structure was formed on the surface, but just small clusters [Figs. 1(c) and 1(d)]. Since they do not follow the underlying symmetry, they are considered as amorphous. In contrast, triangular islands were grown on the surface for the substrate temperature at $200-250{ }^{\circ} \mathrm{C}$ [Figs. 1(e) and 1(f)], whose atomic structure is similar to that of the $\mathrm{Si}(311)-\mathrm{In}-3 \times 2$ surface. The distribution of the size of the triangular island measured from the STM image is shown in the inset in Fig. 1(e). Furthermore, when the temperature was kept at $50-150{ }^{\circ} \mathrm{C}$, a single nanowire was formed along the upper edge of the triangular island in the [110] direction [Figs. 1(g) and 1(h)]. The edge of the triangular structure here is considered to function as a barrier that traps In atoms, promoting the formation of In nanowires. ${ }^{14,15}$ To grow In nanowires, a high In deposition rate $(\sim 2.0 \mathrm{ML} / \mathrm{min})$ was needed with the optimization of the substrate temperature. As the inset in Fig. 1(g) shows, the distribution is narrower than that in Fig. 1(e).

Second, we represent the method of forming In nanodots on a Si surface. With the deposition of 0.6 ML of In atoms on a clean $\mathrm{Si}(111)-7 \times 7$ surface at $300{ }^{\circ} \mathrm{C}$, a uniform $\mathrm{Si}(111)-\mathrm{In}-\sqrt{31} \times \sqrt{31}$ structure is formed, ${ }^{16}$ which we used as the substrate. When In atoms were deposited with the substrate temperature being kept at RT, In nanodots were grown on the surface, as shown in Fig. 2. Figures 2(a)-2(c) show the STM images obtained for the nanodots grown by depositions of about $0.1,0.2$, and $0.3 \mathrm{ML}$ of In, respectively. From the diameter distributions of the nanodots shown in the insets, the size of the nanodots increases with deposition ra- 

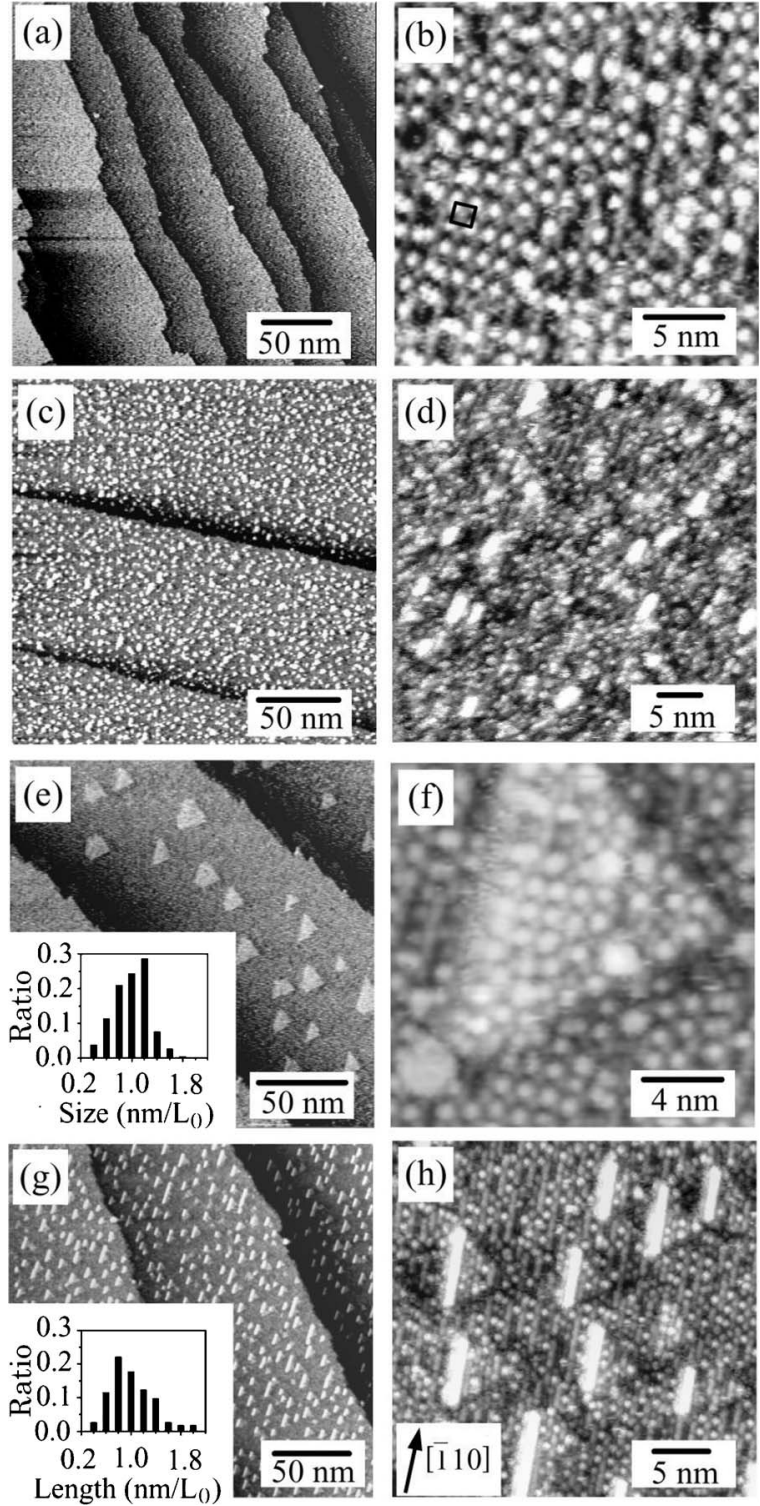

FIG. 1. STM images obtained for the large and small areas of (a) and (b) $\mathrm{Si}(311)-\mathrm{In}-3 \times 2$ (substrate temperature $T_{s}=\sim 300^{\circ} \mathrm{C}, 1-15 \mathrm{ML}$ : large islands are also formed at high coverage, $V_{s}=-2.0 \mathrm{~V}, I_{t}=0.2 \mathrm{nA}$ ), [(c) and (d)] small In clusters $\left(T_{s}=\sim 50{ }^{\circ} \mathrm{C}, 1 \mathrm{ML}, V_{s}=2.0 \mathrm{~V}, I_{t}=0.2 \mathrm{nA}\right),[(\mathrm{e})$ and (f) ] triangular structures $\left(T_{s}=200-250{ }^{\circ} \mathrm{C}, 1 \mathrm{ML}, V_{s}=-2.0 \mathrm{~V}, I_{t}=0.2 \mathrm{nA}\right)$, and $[(\mathrm{g})$ and $(\mathrm{h})]$ triangular structures with nanowires $\left(T_{s}=50-150{ }^{\circ} \mathrm{C}\right.$, $\left.1 \mathrm{ML}, V_{s}=-2.0 \mathrm{~V}, I_{t}=0.2 \mathrm{nA}\right)$. The insets in (e) and (g) show the distributions of the triangular size (length of side without wire) and the wire length reduced by the averaged lengths of $L_{0}(16$ and $8.1 \mathrm{~nm})$, respectively.

tio, but their density remains almost constant, as shown in Fig. 2. Remarkably, the size distribution of the nanodots becomes narrow with In deposition [Fig. 2(d)], which is attributed to the kinetically self-limiting effect and/or thermodynamic effect of strain-induced energy minimization. ${ }^{1}$ This is the demonstration of the formation of well-shaped regular nanodots with the diameter of a few nanometers for an $\mathrm{In} / \mathrm{Si}$ surface. ${ }^{17}$

Third, a selective formation of nanowires on a $\mathrm{Si}(111)-\mathrm{In}-4 \times 1$ structure is demonstrated. The $\mathrm{Si}(111)-\mathrm{In}-4 \times 1$ surface has been extensively studied to reveal its atomic structure and electronic properties. ${ }^{7,18-21}$ However, the wire array of the $\operatorname{Si}(111)-\mathrm{In}-4 \times 1$ surface is uniform; despite the interest in the formation of threedimensional In nanowires and the theoretical studies of the
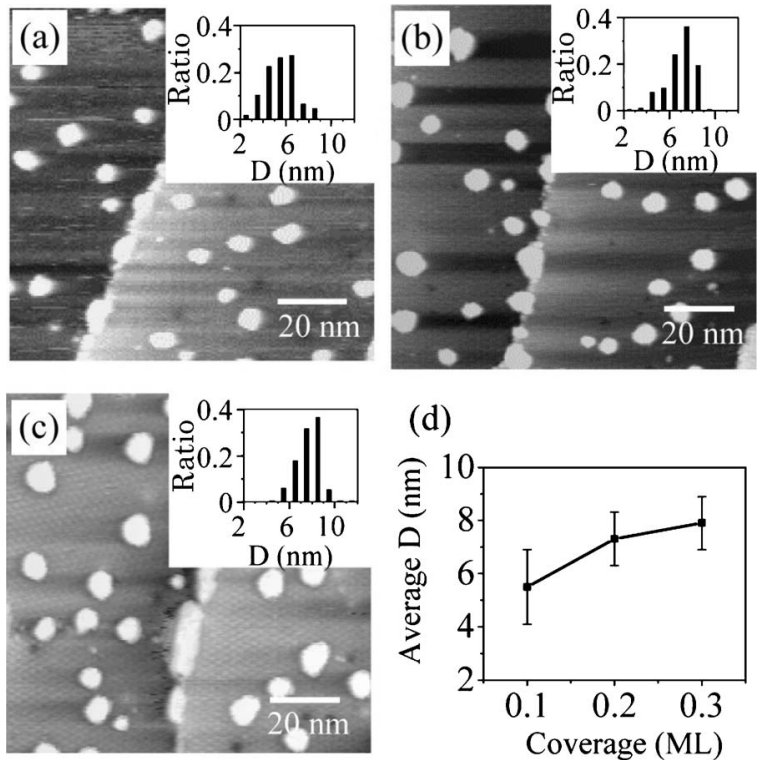

(d)

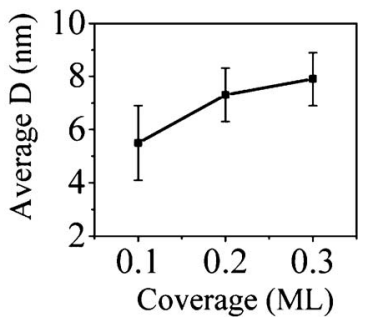

FIG. 2. STM images of In nanodots grown at RT with In coverages of (a) $0.1 \mathrm{ML}\left(V_{s}=-1.0 \mathrm{~V}, I_{t}=0.2 \mathrm{nA}\right),(\mathrm{b}) 0.2 \mathrm{ML},\left(V_{s}=-2.0 \mathrm{~V}, I_{t}=0.5 \mathrm{nA}\right)$, and (c) $0.3 \mathrm{ML}\left(V_{s}=-1.0 \mathrm{~V}, \quad I_{t}=0.2 \mathrm{nA}\right)$. The diameter (d) distribution of In dots is shown in the inset, the average value of which is shown in (d) as a function of coverage.

structure, it is still a challenging issue to experimentally grow In wire arrays on a homogeneous In $-4 \times 1$ surface because of the fast diffusion of In adatoms on the surface even at RT.

Here, we realized the formation of In nanowires on the In- $4 \times 1$ surface with the combination of the RT growth and use of an $\operatorname{In} / \mathrm{Si}(111)-4 \times 1 / \sqrt{31} \times \sqrt{31}$ coexisting phase as the substrate. Figure 3(a) shows an STM image of the template surface grown by the deposition of $\sim 0.7 \mathrm{ML}$ of In on a Si(111)- $(7 \times 7)$ surface at $\sim 400{ }^{\circ}$ C. As shown in Fig. 3(a), the surface consists of two phases with the $4 \times 1$ and $\sqrt{31}$ $\times \sqrt{31}$ structures. The In coverage of the $\sqrt{31} \times \sqrt{31}$ phase is about $0.6 \mathrm{ML},{ }^{16}$ while that of the $4 \times 1$ phase is $1 \mathrm{ML}^{14}$ Therefore, the density and size of the $4 \times 1$ and $\sqrt{31} \times \sqrt{31}$ phase areas can be controlled by adjusting the substrate temperature and In coverage from 0.6 to 1.0 ML. After the structure with the two phases at a certain ratio was formed, the sample was cooled to RT, and In atoms were deposited.

Figure 3(b) shows an STM image obtained after the deposition of $\sim 0.2 \mathrm{ML}$ of In on the template surface shown in Fig. 1(a). As was observed in Fig. 2, In nanodots are formed on the $\sqrt{31} \times \sqrt{31}$ phase that surrounds the $4 \times 1$ phase area. However, In nanowires are selectively formed in the In- $(4 \times 1)$ area. Since no wires but large islands were formed on a homogeneous $4 \times 1$ surface without the $\sqrt{31}$ $\times \sqrt{31}$ phase at RT, the boundary barrier may play an important role in the nucleation and growth of the In wires on a $\operatorname{Si}(111)-\operatorname{In}-4 \times 1 / \sqrt{31} \times \sqrt{31}$ surface. Similar boundary effect may work in part in the case shown in Figs. 1(g) and 1(h). To clarify the detailed mechanism, further study is necessary.

Figures 3(c)-3(f) show the STM images of the nanowire arrays obtained for three different In coverages and their cross sections. The wire array of $0.1 \mathrm{ML}$ In coverage shows a wide spacing. With increasing In coverage to $0.2 \mathrm{ML}$, the space becomes uniform with an eight times periodicity in the 

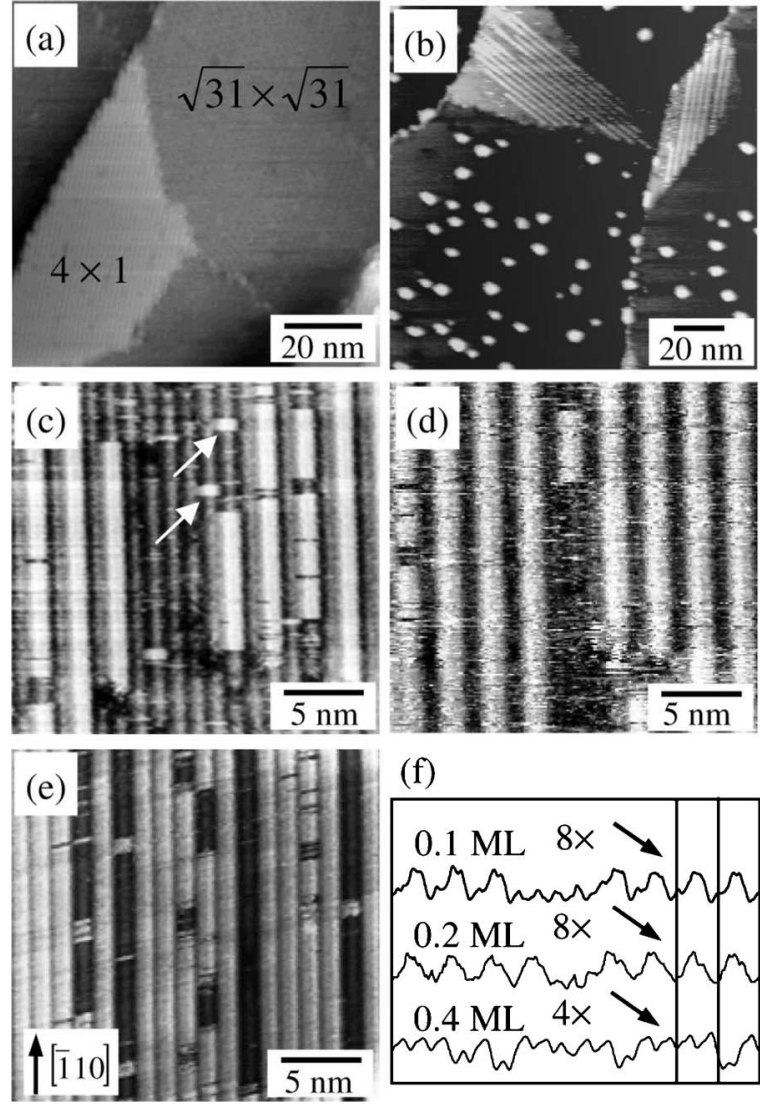

(f)

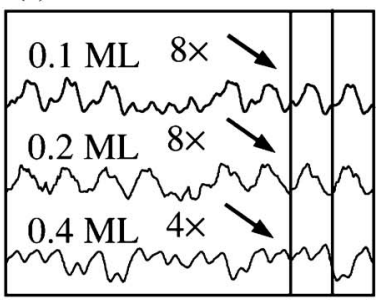

FIG. 3. STM images of (a) In/Si(111) surface with coexisting $4 \times 1$ and $\sqrt{31} \times \sqrt{31}$ phases $\left(0.7 \mathrm{ML}, V_{s}=-1.4 \mathrm{~V}, I_{t}=0.2 \mathrm{nA}\right)$. (b) In nanowires and nanodots formed after the growth of $0.2 \mathrm{ML}$ of In at RT on the template surface of (a) $\left(V_{s}=-0.6 \mathrm{~V}, \quad I_{t}=1.0 \mathrm{nA}\right)$. STM images of nanowire arrays formed on the $4 \times 1$ phase with In coverages of (c) $0.1 \mathrm{ML}$ $\left(V_{s}=-1.0 \mathrm{~V}, \quad I_{t}=0.5 \mathrm{nA}\right),(\mathrm{d}) 0.2 \mathrm{ML}\left(V_{s}=-1.5 \mathrm{~V}, \quad I_{t}=0.3 \mathrm{nA}\right)$, and (e) $0.4 \mathrm{ML}\left(V_{s}=-1.0 \mathrm{~V}, \quad I_{t}=0.2 \mathrm{nA}\right)$. The white arrows in (c) indicate the In adatoms adsorbed on top of substrate In chains and the black arrow in (e) indicates the wire orientation. (f) Cross sections of the wire arrays in (c)-(e).

[112] direction. Then the wire array exhibits a four times periodicity for the growth of $0.4 \mathrm{ML}$ of In. These observations suggest the existence of an interaction between nanowires. This surface consists of wires formed by two zigzag In chains separated by a $\mathrm{Si}$ chain on the almost undistorted $\mathrm{Si}$ substrate. ${ }^{19}$ It is theoretically predicted that In adatoms prefer to adsorb on the sites between two neighboring In wires, which yields a positional shift in the In atoms in the chains. ${ }^{22}$ As indicated by white arrows in Fig. 3(c), the In atoms are adsorbed on the ridge of the substrate wires. The induced lattice deformation results in a local strain and may cause the observed interaction between the nanowires. With this method, the sizes and the ratio of the areas with nanodots and nanowires can be controlled. The interaction may produce new properties, such as a change in critical temperature. ${ }^{23}$

In summary, methods of forming various In nanostructures on Si surfaces are demonstrated. A variety of nanoscale elements will enable further development in designing and controlling nanoscale electronic and optical devices. The findings are expected to make the system more applicable to future semiconductor technologies as well as to fundamental research studies. Studies on the detailed atomic structures and electronic properties of the nanostructures are in progress.

We thank Ms. Rie Yamashita in our group at University of Tsukuba for her help in preparing this paper.

${ }^{1}$ V. A. Shchukin and D. Bimberg, Rev. Mod. Phys. 71, 1125 (1999).

${ }^{2}$ P. Moriarty, Rep. Prog. Phys. 64, 297 (2001).

${ }^{3}$ F. Rosei, J. Phys.: Condens. Matter 16, S1373 (2004).

${ }^{4}$ H. Brune, Surf. Sci. Rep. 31, 121 (1998).

${ }^{5}$ Z. Gai, G. A. Farnan, J. P. Pierce, and J. Shen, Appl. Phys. Lett. 81, 742 (2002).

${ }^{6}$ C. Teichert, J. Barthel, H. P. Oepen, and J. Kirschner, Appl. Phys. Lett. 74, 588 (1999).

${ }^{7}$ H. W. Yeom, S. Takeda, E. Rotenberg, I. Matsuda, K. Horikoshi, J. Schaefer, C. M. Lee, S. D. Kevan, T. Ohta, T. Nagao, and S. Hasegawa, Phys. Rev. Lett. 82, 4898 (1999).

${ }^{8}$ Y. Terada, S. Yoshida, A. Okubo, K. Kanazawa, M. Xu, O. Takeuchi, and H. Shigekawa, Nano Lett. 8, 3577 (2008).

${ }^{9}$ M. Hupalo and M. C. Tringides, Phys. Rev. B 73, 041405(R) (2006).

${ }^{10}$ C. Liu, T. Uchihashi, and T. Nakayama, Phys. Rev. Lett. 101, 146104 (2008).

${ }^{11}$ T. Uchihashi, C. Ohbuchi, S. Tsukamoto, and T. Nakayama, Phys. Rev. Lett. 96, 136104 (2006).

${ }^{12}$ J. Dabrowski, H. J. Müssig, and G. Wolff, Phys. Rev. Lett. 73, 1660 (1994).

${ }^{13}$ H. Hibino and M. C. Tringides, Phys. Rev. B 56, 4092 (1997).

${ }^{14}$ P. Gambardella, M. Blanc, L. Bürgi, K. Kuhnke, and K. Kern, Surf. Sci. 449, 93 (2000).

${ }^{15}$ P. Gambardella, A. Dallmeyer, K. Maiti, M. C. Malagoli, W. Eberhardt, K. Kern, and C. Carbone, Nature (London) 416, 301 (2002).

${ }^{16}$ A. A. Saranin, A. V. Zotov, A. N. Tovpik, M. A. Cherevik, E. N. Chukurov, V. G. Lifshits, M. Katayama, and K. Oura, Surf. Sci. 450, 34 (2000).

${ }^{17}$ M. A. Hafez, M. S. Hegazy, and H. E. Elsayed-Ali, J. Vac. Sci. Technol. A 23, 61681 (2005).

${ }^{18}$ J. J. Lander and J. Morrison, J. Appl. Phys. 36, 1706 (1965).

${ }^{19}$ O. Bunk, G. Falkenberg, J. H. Zeysing, L. Lottermoser, R. L. Johnson, M. Nielsen, F. Berg-Rasmussen, J. Baker, and R. Feidenhans'1, Phys. Rev. B 59, 12228 (1999).

${ }^{20}$ T. Abukawa, M. Sasaki, F. Hisamatsu, T. Goto, T. Kinoshita, A. Kakizaki, and S. Kono, Surf. Sci. 325, 33 (1995).

${ }^{21}$ I. G. Hill and A. B. McLean, Phys. Rev. Lett. 82, 2155 (1999).

${ }^{22}$ S. Wippermann, N. Koch, and W. G. Schmidt, Phys. Rev. Lett. 100, 106802 (2008).

${ }^{23}$ S. Kurata and T. Yokoyama, Phys. Rev. B 71, 121306(R) (2005). 